\title{
PTMProphet: Fast and Accurate Mass Modification Localization for the Trans-Proteomic Pipeline
}

David D. Shteynberg ${ }^{1}$, Eric W. Deutsch ${ }^{1}{ }^{*}$, David S. Campbell ${ }^{1}$, Michael R. Hoopmann ${ }^{1}$, Ulrike Kusebauch ${ }^{1}$, Dave Lee ${ }^{2}$, Luis Mendoza ${ }^{1}$, Mukul K. Midha ${ }^{1}$, Zhi Sun ${ }^{1}$, Anthony D. Whetton ${ }^{2}$, and Robert L. Moritz ${ }^{1}$

${ }^{1}$ Institute for Systems Biology, Seattle, WA, 98008, USA

2 Stoller Biomarker Discovery Centre, University of Manchester, Manchester, M13 9PL, UK

*Address correspondence to: Eric W. Deutsch, Institute for Systems Biology, 401 Terry Ave N, Seattle, WA 98109, USA, Email: edeutsch@systemsbiology.org, Phone: 206-732-1200, Fax: 206-732-1299

\section{Synthetic phosphopeptides (example data set 2)}

1342 phosphorylated peptides were individually chemically synthesized as free amine at the $\mathrm{N}$ terminus and carboxylic acid at the C-terminus (JPT Peptide Technologies, Micro-scale Peptides, $50 \mathrm{nmol}$ per peptide, crude). Phosphorylated serine, threonine and tyrosine were introduced as phosphorylated building block and cysteine residues as carboxyamidomethylated cysteine building block.

Peptides were analyzed in pools of maximum 95 peptides on a SCIEX TripleTOF® 5600+ equipped with a Nanospray-III® Source (Sciex) and an Ekspert ${ }^{\mathrm{TM}}$ nanoLC 425 with $\mathrm{cHiPLC} \circledast$ system operated in trap-elute mode (Eksigent). Peptides were loaded on a cHiPLC trap (200 $\mu \mathrm{m}$ x $500 \mu \mathrm{m}$ ChromXP C18-CL, $3 \mu \mathrm{m}, 120 \AA$ ) and washed for 10 minutes at $2 \mu \mathrm{L} / \mathrm{min}$. Peptides were eluted on a nano cHiPLC column (75 $\mu \mathrm{m} \times 15 \mathrm{~cm}$ ChromXP C18-CL, $3 \mu \mathrm{m}, 120 \AA$ ) with $0.1 \%$ formic acid in water (A), $0.1 \%$ formic acid in acetonitrile $(B)(v / v)$ at $300 \mathrm{~nL} / \mathrm{min}$ using a gradient from $3 \%$ to $43 \% \mathrm{~B}$ in $80 \mathrm{~min}, 43 \%-63 \% \mathrm{~B}$ at $80-85 \mathrm{~min}$ and $63 \%-83 \% \mathrm{~B}$ at $85-87 \mathrm{~min}$ at a flow 
rate of $300 \mathrm{~nL} / \mathrm{min}$. A survey scan (TOF-MS) was acquired in the $\mathrm{m} / \mathrm{z}$ range of $400-1,250$ Da with 250 msec accumulation time. The 30 most intense precursor ions with charge state 2-5 were selected for fragmentation with rolling collision energy. MS/MS fragment spectra were collected in the range of $100-1,500 \mathrm{Da}$ with $50 \mathrm{msec}$ accumulation and a $1.8 \mathrm{~s}$ period cycle time. Former target ions were excluded for 15 seconds. 